\title{
RESPIRATORY SYNCYTIAL VIRUS INFECTION DOWNREGULATES ANTIOXIDANT ENZYME EXPRESSION BY TRIGGERING DEACETYLATION- PROTEASOMAL DEGRADATION OF NRF2
}

Narayana Komaravelli ${ }^{1}$, Bing Tian $^{2}$, Teodora Ivanciuc ${ }^{1}$, Nicholas Mautemps ${ }^{1}$, Allan R. Brasier $^{2,3}$, Roberto P. Garofalo ${ }^{1,3}$, and Antonella Casola ${ }^{1,3^{*}}$

Departments of Pediatrics $^{1}$, Internal Medicine ${ }^{2}$, and Sealy Center for Molecular Medicine ${ }^{3}$, University of Texas Medical Branch at Galveston, Texas, 77555

*Correspondence should be addressed to: Antonella Casola, M.D., Department of Pediatrics, 301 University Blvd., Galveston, TX, 77555-0372; Fax (409) 772-1761; Tel. (409) 747-0581; Email: ancasola@utmb.edu 


\section{ABBREVIATIONS}

\begin{tabular}{|c|c|}
\hline AEC & Airway epithelial cell \\
\hline $\mathrm{AOE}$ & Antioxidant enzyme \\
\hline ARE & Antioxidant response element \\
\hline BHA & Butylated hydroxyanisole \\
\hline ChIP & Chromatin immunoprecipitation \\
\hline GCLC & glutamyl cysteine ligase-catalytic \\
\hline GPX1 & Glutathione peroxidase 1 \\
\hline HDAC & Histone deacetylase \\
\hline IP & Immunoprecipitation \\
\hline KEAP1 & Kelch like-ECH-associated protein 1 \\
\hline NQO1 & NAD(P)H:quinone oxidoreductase 1 \\
\hline NRF2 & Nuclear factor erythroid 2-related factor 2 \\
\hline QgPCR & Quantitative genomic PCR \\
\hline ROS & Reactive oxygen species \\
\hline RSV & Respiratory syncytial virus \\
\hline SAEC & Small airway epithelial cell \\
\hline SOD1 & Superoxide dismutase 1 \\
\hline $\mathrm{tBHQ}$ & t-Butylhydroquinone \\
\hline TSA & Trichostatin A \\
\hline $\mathrm{Ub}$ & Ubiquitin \\
\hline
\end{tabular}




\section{ABSTRACT}

Respiratory syncytial virus (RSV) is the most important cause of viral acute respiratory tract infections and hospitalizations in children, for which no vaccine or treatment is available. RSV infection in cells, mice and children leads to rapid generation of reactive oxygen species, which are associated with oxidative stress and lung damage, due to a significant decrease in the expression of airway antioxidant enzymes (AOEs). Oxidative stress plays an important role in the pathogenesis of RSV-induced lung disease, as antioxidants ameliorate clinical disease and inflammation in vivo. The aim of this study is to investigate the unknown mechanism(s) of viralinduced inhibition of AOE expression. This study shows that RSV infection induces a progressive reduction in nuclear and total cellular level of the transcription factor NF-E2-related factor 2 (NRF2), resulting in decreased binding to endogenous AOE gene promoters and decreased AOE expression. RSV induces NRF2 deacetylation and degradation via the proteasome pathway in vitro and in vivo. Histone deacetylase and proteasome inhibitors block NRF2 degradation and increase NRF2 binding to AOE endogenous promoters, resulting in increased AOE expression. Known inducers of NRF2 are able to increase NRF2 activation and subsequent AOE expression during RSV infection in vitro and in vivo, with significant amelioration of oxidative stress. This is the first study to investigate the mechanism(s) of viralinduced inhibition of AOE expression. RSV-induced inhibition of NRF2 activation, due to deacetylation and proteasomal degradation, could be targeted for therapeutic intervention aimed to increase antioxidant airway capacity during infection.

Keywords: Respiratory Syncytial Virus, ROS, oxidative stress, antioxidant enzymes, NRF2, acetylation, proteasome 


\section{Running title: RSV and NRF2}

\section{INTRODUCTION}

Respiratory syncytial virus (RSV) is the single most important virus causing acute respiratory tract infections in children, with an estimated $40-90 \%$ of children with bronchiolitis and $25-50 \%$ of children with viral pneumonia infected with RSV [1]. It is also a major cause of severe respiratory morbidity and mortality in the elderly [2], being responsible for 64 million clinical infections and 160 thousand deaths annually worldwide [3]. In addition to acute morbidity, RSV infection has been linked to both the development and the severity of asthma. No vaccine or effective treatment is currently available for RSV. In a series of in vitro and in vivo studies, over the past few years, we have discovered that in the course of RSV infection, reactive oxygen species (ROS) are rapidly generated and they are associated with cellular oxidative damage, indicated by an increase in lipid peroxidation, lung inflammation and clinical disease [4-6]. RSV-induced ROS formation also controls inducible expression of chemokine and other inflammatory genes in response to infection $[7,8]$. Antioxidant treatment significantly ameliorates RSV-induced clinical disease and pulmonary inflammation in a mouse model of infection, suggesting a casual relationship between increased ROS production and lung disease [6]. We found that the expression and/or activity of the antioxidant enzymes (AOEs) superoxide dismutase (SOD), catalase, glutathione peroxidase (GPx) and glutathione S-transferase (GST) were dramatically decreased in RSV-infected human airway epithelial cells (AECs)[4]. Similar decreases in AOE expression were also observed in the lungs of RSV-infected mice and in nasopharyngeal secretions (NPS) of children with severe RSV-induced lower respiratory tract infections [5], suggesting that oxidative damage associated with RSV infection results from an 
imbalance between ROS production and antioxidant cellular defenses. Transcription of many oxidative stress-inducible genes is regulated in part through cis-acting antioxidant responsive element (ARE) sequences. This element has been identified in the regulatory regions of genes encoding detoxification enzymes, such as NQO1 (NADPH:quinone oxidoreductase), as well as many AOEs, including SOD1, catalase, heme oxygenase 1, GST and glutathione-generating enzymes such as glutamate cysteine ligase (GCLC) (Reviewed in [9]). NF-E2-related factor 2 (NRF2) is an important redox-responsive protein that helps protect the cells from oxidative stress and injury (Reviewed in [10]). It is a basic leucine zipper transcription factor that is normally bound in the cytosol to a cytoskeleton-associated inhibitor called KEAP1 (Kelch-like-ECH associated protein 1). Electrophile-induced release of NRF2 is proposed to involve covalent modifications of KEAP1 and/or NRF2 in the cytoplasm. Such modifications include oxidation of key cysteine residues in KEAP1, phosphorylation of NRF2, and switching of Cullin-3-dependent ubiquitination from NRF2 to KEAP1, leading to the degradation of KEAP1 and stabilization and activation of NRF2. The released NRF2 then translocates to the nucleus and binds to ARE sites to promote gene transcription [10]. During activation, NRF2 also undergoes different type of post-translational modifications, including phosphorylation, which regulates nuclear translocation and export [11], as well as acetylation, which is important for stabilization of NRF2 binding to DNA once activated [12].

The aim of our study was to investigate the unexplored mechanism(s) leading to viralinduced decreased expression of AOE. Our data show that RSV infection induces a progressive reduction in nuclear and total cellular levels of the NRF2, resulting in decreased binding to ARE site of endogenous AOE gene promoter, with subsequent decrease in their expression. RSV induces NRF2 deacetylation, ubiquitination, and degradation via the proteasome pathway both in 
vitro and in vivo. Histone deacetylase (HDAC) and proteasome inhibitors block NRF2 degradation and increase NRF2 binding to endogenous promoter ARE sites, resulting in increased AOE expression. Known inducers of NRF2 are able to increase NRF2 activation and subsequent AOE expression during RSV infection in AECs, as well as in an animal model of infection, with significant amelioration of oxidative stress, which is an important pathogenic component of viral-induced lung disease, adding additional support to the concept that therapeutic strategies aimed to increase antioxidant airway capacity by increasing NRF2 activity could be beneficial in RSV infection.

\section{MATERIALS AND METHODS}

\section{Materials}

BHA, tBHQ and TSA were purchased from Sigma, MO, USA. MG132 and Lactacystin were purchased from Calbiochem, CA, USA.

\section{$R S V$ preparation}

The RSV Long strain was grown in Hep- 2 cells and purified by centrifugation on discontinuous sucrose gradients as described elsewhere [13]. The virus titer of the purified RSV pools was 8-9 $\log _{10}$ plaque forming units $(\mathrm{PFU}) / \mathrm{mL}$ using a methylcellulose plaque assay. No contaminating cytokines were found in these sucrose-purified viral preparations [14]. LPS, assayed using the limulus hemocyanin agglutination assay, was not detected. Virus pools were aliquoted, quickfrozen on dry ice/alcohol and stored at $-80^{\circ} \mathrm{C}$ until used.

\section{Cell Culture and Infection of Epithelial Cells with RSV}


A549 cells, a human alveolar type II like epithelial cell line (American Type Culture Collection, Manassas, VA) and small alveolar epithelial cells (SAECs) (from Clonetics, now part of Lonza Inc., San Diego, CA), normal human AECs derived from terminal bronchioli, were grown according to the manufacturer's instructions. RSV infection in A549 cells were done in F12K medium containing $2 \%$ FBS. When SAECs were used for RSV infection, they were changed to basal medium, not supplemented with growth factors, 6 hours before and throughout the length of the experiment. At 80 to $90 \%$ confluence, cell monolayers were infected with RSV at multiplicity of infection (MOI) of 3. An equivalent amount of 30\% sucrose solution was added to uninfected A549 and SAECs, as a control.

For tBHQ and TSA experiments, cells were pretreated with the compounds for 1 hour and then infected in their presence for the duration of the experiment. In selected experiments, tBHQ was also added at different time points after infection. For proteasome inhibitors experiments, MG132 or Lactacystin were added 10h post-infection (p.i.). Equal amounts of diluent were added to cells uninfected and infected as control. Total number of cells and cell viability, following various treatments, were measured by trypan blue exclusion. There was no significant change in cell viability with all compounds tested. Similarly, there was no effect of both compounds on viral replication, tested by plaque assay.

\section{Reporter gene assay}

Logarithmically growing A549 cells were transfected in triplicate with Cignal Antioxidant Response Reporter from Qiagen, (Cat \# 336841, Maryland), an optimized luciferase reporter construct containing the NQO1 ARE promoter sequence, together with $\beta$-galactosidase expression plasmid. Cells were infected with RSV in the presence or absence of specific 
inhibitors and harvested at $24 \mathrm{~h}$ p.i. to independently measure luciferase and $\beta$-galactosidase reporter activity, as previously described [15]. Luciferase activity was normalized to the internal control $\beta$-galactosidase activity. Results are expressed in arbitrary units.

\section{Western blot}

Nuclear extracts of uninfected and infected cells were prepared using hypotonic/nonionic detergent lysis, according to Schaffner protocol [16]. To prevent contamination with cytoplasmic proteins, isolated nuclei were purified by centrifugation through $1.7 \mathrm{M}$ sucrose buffer A for 30 minutes, at 12,000 rpm, before nuclear protein extraction, as previously described $[16,17]$. Total cell lysates of uninfected and infected cells were prepared as previously described [16]. Equal amount of proteins (10 to $20 \mu \mathrm{g}$ ) were separated by SDS-PAGE and transferred onto polyvinylidene difluoride membrane. Nonspecific binding was blocked by immersing the membrane in Tris-buffered saline-Tween (TBST) blocking solution containing 5\% skim milk powder. After a short wash in TBST, the membranes were incubated with the primary antibody overnight at $4{ }^{\circ} \mathrm{C}$, followed by the appropriate secondary antibody diluted in TBST for 1 hour at room temperature. Proteins were detected using enhancedchemiluminescence assay. Densitometric analysis of band intensities was performed using UVP VisionWorksLS Image Acquisition and Analysis Software 8.0 RC 1.2 (UVP, Upland, CA). The

primary antibodies used for Western blots were anti-NRF2 (H-300, sc-13032), anti HDAC1(H51, sc-7872), -HDAC2 (H-54, sc-7899), -HDAC3 (H-99, sc-11417) from Santa Cruz Biotechnology Inc, CA, anti-SOD1 (SOD100, Stressgen Bioreagents, MI), anti-lamin B (GWB5CD4D4, GenWay Biotech) and anti- $\beta$-Actin (A1978 Sigma, MO). 


\section{Immunoprecipitation}

$250 \mu \mathrm{g}$ of total cell lysate or $200 \mu \mathrm{g}$ of nuclear extracts from RSV-infected A549 or SAECs were immunoprecipitated using $5 \mu \mathrm{g}$ of anti-NRF2 antibody and protein A/G agarose beads (Santa Cruz Biotechnology Inc, sc-2003). Complexes were eluted in 2x SDS PAGE buffer and subjected to Western blot analysis using anti-ubiquitin (SC-8017, Santa Cruz Biotechnology Inc, CA) or anti-acetyl lysine (ab21623, Abcam, MA) antibodies.

\section{HDAC Activity}

Nuclear extracts prepared from A549 cells and SAECs uninfected or infected with RSV were assayed for HDAC activity using a commercially available kit (10011563, Cayman, Ann Arbor, MI ) according to the manufacturer's instructions.

\section{8-Isoprostane assay}

Measurements of $\mathrm{F}_{2} 8$-isoprostane was performed using a competitive enzyme immunoassay from Cayman Chemical (Cat 516351, Ann Arbor, MI).

\section{Quantitative Reverse Transcriptase PCR (Q-RT-PCR)}

Total RNA was extracted using ToTALLY RNA kit from Ambion (Cat \# AM1910, Austin, TX). RNA samples were quantified using a Nanodrop Spectrophotometer (Nanodrop Technologies) and quality was analyzed using the Agilent 2100 Bioanalyzer (Agilent Technologies, Santa Clara, CA. Synthesis of cDNA was performed with 1ug of total RNA in a 20ul reaction using the reagents in the Taqman Reverse Transcription Reagents Kit from ABI (Applied Biosystems \#N8080234), according to manufacturer's instructions. Q-PCR amplification was done using 
$1 \mu 1$ of cDNA in a total volume of $25 \mu 1$ using the Faststart Universal SYBR green Master Mix (Roche Applied Science \#04913850001). The final concentration of the primers was 300nM. 18S RNA was used as housekeeping gene for normalization. PCR assays were run in the ABI Prism 7500 Sequence Detection System. Duplicate CT values were analyzed in Microsoft Excel using the comparative $\mathrm{CT}(\Delta \Delta \mathrm{CT})$ method as described by the manufacturer (Applied Biosystems). The amount of target $\left(2^{-\Delta \Delta C T}\right)$ was obtained by normalizing to endogenous reference (18S) sample. Primer sequences are available upon request.

Two-step Chromatin immunoprecipitation (XChIP) and quantitative genomic PCR (Q-gPCR) For XChIP we used ChIP-IT Express kit from Active Motif (Cat \# 53008 \& 53032, Carlsbad, CA) and followed manufacturer instruction with slight modification. Briefly, A549 cells in 10 $\mathrm{cm}$ plate were washed three times with PBS and fixed with freshly prepared $2 \mathrm{mM}$ disuccinimidyl gluterate (DSG) (Cat \# 20593, Thermo Scientific, Rockford, IL). After three washes with PBS, cells were fixed with freshly prepared formaldehyde for $1 \mathrm{~min}$ and neutralized with glycine for 5 min at room temperature. Cells were harvested and disrupted using a dounce homogenizer to isolate nuclei. Nuclei were sheared by sonication to obtain DNA fragments from 200 to 1500 base pair (bp). 20 micrograms of sheared chromatin were immunoprecipitated with $5 \mu \mathrm{g}$ of ChIP grade anti-NRF2 (sc-722X), -CBP (sc-369X), -or HDAC1 (sc-7872X) antibody from Santa Cruz Biotechnology, CA, USA, and magnetic beads conjugated with protein $\mathrm{G}$ at $4^{\circ} \mathrm{C}$ overnight. Immunoprecipitation with IgG antibody was used as negative control. Chromatin was reverse crosslinked, eluted from magnetic beads, and purified using PCR purification kit (Cat \# 28106, Qiagen GmbH, Hilden). Q-gPCR was done by SyBR green based real time PCR using the following primers spanning the SOD1 gene promoter ARE site: forward- 
AAAGCATCCATCTTGGGGCG and reverse- AACCTTCTTTTCACGGGGGC, or the catalase promoter ARE site: forward-AACGGCCGCGTCCCAG and reverse-

CTCTCCGAAGGAGGCCTGAA. Total input chromatin DNA for immunoprecipitation was included as positive control for PCR amplification.

In vivo studies

10-12 weeks old female BALB/c mice were purchased from Harlan (Houston, TX) and were housed in pathogen-free conditions in the animal research facility of the University Texas Medical Branch (UTMB), Galveston, Texas, in accordance with the National Institutes of Health and UTMB institutional guidelines for animal care. Experiments were performed with a minimum of four to six animals/group. Under light anesthesia, mice were inoculated intranasally with $10^{7} \mathrm{PFU}$ of sucrose-purified RSV (Long Strain) in a final volume of $50 \mu 1 /$ dose diluted in phosphate buffered saline (PBS). Control animals (mock infected, defined as sham) received PBS treated in a similar manner. Mice were treated by gavage with $250 \mathrm{mg} / \mathrm{kg}$ body weight of BHA or corn oil (diluents for BHA) 2 days prior RSV infection and during the first 2 days of infection. Bronchoalveolar lavages were prepared by flushing the lungs twice via the trachea with $1 \mathrm{~mL}$ of ice-cold PBS. BAL fluid supernatant were collected at 48 hours post-infection following centrifugation ( $5 \mathrm{~min}$ at $5000 \mathrm{rpm}$ ), and stored at $-80^{\circ} \mathrm{C}$ prior to 8 -isoprostane assay. Lungs samples from all groups were harvested at 48 hours p.i. to assess mRNA levels of SOD1 and catalase by Q-RT-PCR, and NRF2 nuclear levels by Western blot. Mice were given i.n. proteasome inhibitor MG-132 at 10 $\mathrm{g} / \mathrm{dose}$, or an appropriate volume of vehicle, 1 hour before infection. Lungs samples from all groups were harvested at 48 hours p.i. to assess mRNA levels of SOD1 and catalase by Q-RT-PCR, and NRF2 nuclear levels by Western blot. 


\section{Statistical analysis}

All results are expressed as mean \pm SEM. Data were analyzed using the GraphPad Prism 5 software. Results were compared among treatment groups by either one-way ANOVA analysis followed by Tukey's post-hoc test or two-way ANOVA analysis followed by Bonferroni posttest. Significance was accepted at $p<0.05$. To streamline figures, all significant results were reported as $\mathrm{p}<0.05$, although in many instances it was well below that threshold.

\section{RESULTS}

\section{RSV infection downregulates NRF2-dependent gene transcription}

To determine whether NRF2 activation was affected in response to RSV infection, nuclear proteins isolated from A549 cells infected for various length of time were subjected to Western blot analysis. After an initial modest increase in nuclear translocation, around $6 \mathrm{~h}$ p.i., there was a progressive, time-dependent decrease in NRF2 nuclear amounts in infected cells at $15 \mathrm{~h}$ and later to levels below that of uninfected cells (Figure 1A, left panel). To confirm our findings in A549 cells, which are a widely accepted model for studying RSV-epithelial cell interactions of the lower airways, a similar experiment was performed in SAECs, normal human airway epithelial cells derived from bronchioli, which represents a major target of RSV infection, as shown in post-mortem studies by us [18] and others [19]. In SAECs infected with RSV we observed an identical response, associated with decreased NRF2 nuclear levels at 15 and 24h p.i. (Figure 1A, right panel). The reduction in nuclear translocation was associated with reduced NRF2dependent gene transcription, demonstrated by reporter gene assay. A549 cells were transiently transfected with a synthetic ARE-driven promoter, linked to a luciferase reporter gene, and infected with RSV for 6, 15 and 24h. NRF2-dependent gene transcription increased at $6 \mathrm{~h}$ p.i., but 
then significantly decreased at subsequent time p.i. to values below that of uninfected cells (Figure 1B), correlating with a time dependent decrease in Nrf2 target genes, including NQO1 and the AOEs SOD1, catalase, GPX1 and GCLC (supplementary Figure 1A). To investigate the mechanism, we measured NRF2 occupancy of the ARE sites of SOD1 and catalase by XChIP. NRF2 binding to both promoters was reduced at 15 and 24h p.i., quantitated by Q-gPCR (Figure 1C). A similar result was obtained for the NQO1 gene (supplementary Figure 1B).

In a parallel set of experiments, AECs were treated with a known pro-oxidative stimulus, hydrogen peroxide, to investigate the effect on NRF2 activation. Different from what occurs in the context of RSV infection, hydrogen peroxide induced a sustained increase in NRF2 nuclear levels, up to $15 \mathrm{~h}$ post-treatment, investigated by Western blot analysis, with levels returning to basal conditions by $24 \mathrm{~h}$ (supplementary Figure 2A). The increase in NRF2 activation was paralleled by an increase in NRF2 target genes catalase and SOD1 (supplementary Figure 2B).

\section{RSV infection induces NRF2 degradation}

To determine whether RSV-induced decrease in NRF2 nuclear levels corresponded to a decrease in total cellular levels, whole cell lysates from A549 and SAECs infected with RSV for various lengths of time were subjected to Western blot analysis. Both A549 cells (Figure 2A, left panel) and SAECs (Figure 2A, right panel) showed significantly lower levels of NRF2 at 15 and 24h p.i., compare to uninfected controls and to early time points of infection, suggesting that RSV induces NRF2 degradation, possibly through the proteasome pathway. Treatment of A549 cells with the specific proteasome inhibitor lactacystin rescued NRF2 cellular levels (Figure 2B), indicating that NRF2 degradation associated with RSV infection occurs through the proteasome. To investigate whether RSV induced changes in NRF2 ubiquitination, total cell lysates of either 
A549 cells or SAECs infected with RSV for 6 and 15h were immunoprecipitated with anti-NRF2 antibody and subjected to Western blot analysis using an anti-ubiquitin antibody. RSV infection was associated with increased NRF2 ubiquitination, compared to uninfected cells (Figure 2C), suggesting that this is an important mechanism(s) targeting NRF2 to proteasome degradation.

Treatment of AECs with proteasome inhibitors led not only to increased NRF2 cellular levels, but also rescued NRF2 function, as MG132, another proteasome inhibitor, and lactacystin treatment was associated with increased ARE-dependent gene transcription, shown by reporter gene assays (Figure 3A) and NQO1 and AOE gene expression, analyzed by Q-RT-PCR (Figure 3B), as well as increased NRF2 binding to the endogenous SOD1 and catalase ARE sites, assessed by XChIP (Figure 3C). From these data, we conclude that proteasome inhibition can restore NRF2 expression and function in the context of RSV infection.

\section{RSV infection is associated with NRF2 deacetylation}

Acetylation is a post-translational modification important for stabilization of NRF2 binding to DNA once activated [12]. To determine whether RSV infection could modulate NRF2 acetylation, total cell lysates from A549 cells were immunoprecipitated with anti-NRF2 antibody and subjected to Western blot using anti acetyl-lysine antibody. RSV infection was associated with a significant decrease in basal NRF2 acetylation, both in A549 (Figure 4A, left panel), as well as in SAECs (Figure 4A, right panel), starting as early as 6h p.i. (data shown represent $15 \mathrm{~h}$ p.i.). Treatment of AECs with the HDAC inhibitor Trichostatin A (TSA) significantly restored NRF2 acetylation, leading to increased NRF2 cellular levels as well (Figure 4A, input). RSV infection upregulated nuclear HDAC activity, starting at $6 \mathrm{~h}$ p.i. and continuing up to $24 \mathrm{~h}$ p.i., both in A549 (Figure 4B, left panel), as well as in SAECs (Figure 4B, right panel). In addition, 
RSV infection was associated with a significant reduction of binding of the transacetylase CBP to the ARE site of the SOD1 gene promoter, starting around 15h p.i., after an initial increase in binding at early time points of infection, as determined by XChIP assay (Figure 4C). Increasing CBP expression by transient transfection was able to rescue ARE-driven reporter gene activity in viral-infected cells (Figure 4D), supporting the idea that NRF2 deacetylation could be the result of unbalanced HDAC and acetylation activity.

Inhibition of HDAC activity was able to rescue nuclear levels of NRF2 during viral infection, as shown by Western blot analysis of nuclear fractions from A549 cells (Figure 5A, left panel) and SAECs (Figure 5A, right panel), and importantly, it was also associated with an overall increase in NRF2 cellular levels, assessed by Western blot analysis of total cell lysates from A549 cells (Figure 5B, left panel) and SAECs (Figure 5B, right panel).

Inhibition of HDAC activity was also able to restore ARE-dependent gene transcription, as shown by reporter gene assay in A549 cells transiently transfected with the ARE-driven promoter and infected with RSV in the presence or absence of TSA (Figure 5C), leading to increased expression of NRF2 target genes assessed by Q-RT-PCR, both in A549 (Figure 5D, upper panel, and supplementary Figure 3A) and SAECs (Figure 5D, lower panel, and supplementary Figure 3B). Increased AOE expression was associated with a significant increase of NRF2 occupancy of the catalase and SOD1 promoter ARE sites (Figure 5E), supporting the finding that inhibition of HDAC can rescue NRF2 activation in the context of RSV infection.

\section{HDAC1 and 2 play an important role in RSV-induced inhibition of NRF2 activation}

Human HDACs are classified, based on the sequence similarity and cofactor dependency, into three groups [20]. TSA is broad specific inhibitor and it blocks both Class I \& II HDAC activity 
[21]. As shown before, TSA treatment in AECs infected with RSV infection was able to rescue NRF2 activation. On the other hand, the HDAC class III specific inhibitor Ex-527 [22] did not have a significant effect, indicating that these class of HDAC proteins was not involved in RSVinduced NRF2 inhibition (data not shown). Since HDAC class II proteins are present predominantly in skeletal muscle, heart, brain, and thymus [23, 24], we first investigated the role of HDAC class I proteins, specifically HDAC1, 2 and 3, in RSV-induced NRF2 deacetylation, as they are known to modulate activation of other transcription factors such as Nuclear Factor (NF)$\kappa \mathrm{B}$ and Signal Transducer and Activator of Transcription $[25,26]$. We first determined whether there was any change in HDAC expression. A549 cells were infected with RSV, harvested at different time points after infection to prepare nuclear proteins, and HDAC1, 2 and 3 levels were assessed by Western blot analysis. There was no difference in nuclear levels of any of the three HDAC proteins (supplementary Figure 4), indicating that change in total HDAC activity was not due to an increase in their nuclear amounts. We then inhibited their expression using specific siRNAs. A549 were transfected with either scrambled or siRNAs selectively targeting HDAC1, 2 or 3, infected with RSV, and harvested to prepare either nuclear extracts or total RNA. Western blots analysis showed that NRF2 nuclear levels in HDAC1 and 2 siRNA transfected cells were significantly higher compared to those of scramble transfected ones, following infection with RSV, with HDAC1 siRNA being the most effective in restoring NRF2 activation to levels comparable of that of uninfected cells (Figure 6A and B), while there was no significant change observed in HDAC3 siRNA transfected cells (Figure 6C). In agreement with these findings, mRNA levels of the NRF2 target genes NQO1 and AOEs were significantly higher in RSVinfected cells transfected with siRNA for HDAC1 and 2, but not HDAC3, compared to scrambled (Figure 6D and supplementary Figure 5). To determine whether HDAC1 was binding 
to the ARE site of the SOD1 gene promoter, we performed XChIP/Q-gPCR. HDAC1 occupancy of the SOD1 ARE site was significantly lower in RSV-infected cells at 6h p.i., below levels of uninfected cells, however, it increased significantly at $15 \mathrm{~h}$ p.i. (Figure $6 \mathrm{E}$ ). Treatment of infected cells with TSA resulted in a significant inhibition of HDAC1 recruitment to the SOD1 ARE site (Figure 6E), in agreement with the previously observed changes in NRF2 activation.

\section{NRF2 is deacetylated and degraded through the proteasome in vivo}

To determine whether deacetylation and proteasome degradation played a role in viral-induced inhibition of NRF2 activation in vivo, we performed confirmatory experiments in our mouse model of RSV infection. Nuclear proteins isolated from lungs of mice either sham-inoculated or infected with RSV for $48 \mathrm{~h}$ were tested for HDAC activity and NRF2 acetylation, as described in the in vitro experiments. Similar to our findings in AECs, RSV infection was associated with increased HDAC activity (Figure 7A), as well as a significant decrease in basal NRF2 acetylation, along with reduced NRF2 nuclear levels (Figure 7B).

To determine whether inhibition of proteasome activity could rescue NRF2 expression and ARE-dependent gene expression, mice were treated with MG132 one hour prior to viral infection and harvested to prepare nuclear extract or extract total RNA at $48 \mathrm{~h}$ p.i. Mice infected with RSV and treated with MG132 showed significantly increased NRF2 nuclear levels, compared to untreated infected mice (Figure 7C). Proteasome inhibition was also able to significantly increase SOD1 expression during RSV infection, while there was only a modest rescue of catalase expression (Figure 7D).

NRF2 inducers ameliorate oxidative stress during RSV infection in vitro and in vivo 
Among the compounds known to stimulate ARE-driven transcription [27], butylated hydroxynanisol (BHA) and its metabolite tert-butylhydroquinone (tBHQ) have been shown to increase HO-1, NQO1 and NRF2 protein expression in both primary and cultured cells [28]. Since BHA was effective in decreasing RSV-induced oxidative stress [6], we investigated whether tBHQ treatment could rescue NRF2 activity following viral infection [28]. A549 cells were transiently transfected with the ARE-driven reporter plasmid and infected with RSV in the presence or absence of tBHQ. RSV infection was associated with a significant decrease in reporter gene activity, compared to uninfected cells, which was restored close to levels of uninfected cells by tBHQ treatment (Figure 8A). AOE gene and protein expression, as well as NRF2 nuclear levels, were also significantly increased in RSV-infected cells by tBHQ treatment (Figure 8B, C and supplementary Figure 6A and B), indicating that NRF2 inducers can restore ARE-dependent gene expression following RSV infection. Treatment of AECs with tBHQ up to 6h p.i. was able to restore NRF2 activation and ARE-dependent gene expression in response to RSV infection (supplementary Figure 6C and D), but not at later time points of infection (data not shown). Restoration of AOE cellular capacity was paralleled by a significant reduction of RSV-induced oxidative stress, as shown by a significant decrease of the oxidative marker 8isoprostane in viral-infected, tBHQ-treated cells (Figure 8D). The effect of tBHQ treatment on NRF2 activation was not due to changes in HDAC activity (Figure 8E).

As tBHQ treatment of AECs was able to rescue NRF2 activation, we tested whether BHA (precursor of tBHQ) had a similar effect in the airways of infected mice. Lungs of mice either sham-inoculated or infected with RSV for $48 \mathrm{~h}$ in the presence or absence of BHA (250 $\mathrm{mg} / \mathrm{kg}$ ) were harvested to prepare bronchoalveolar lavages (BALs), nuclear extracts or total RNA. Mice infected with RSV showed significantly reduced NRF2 nuclear levels, compared to 
sham-inoculated mice, and, in most of the infected mice, BHA treatment was able to restore NRF2 activation to levels close to that of uninfected mice (Figure 9A), as well as the expression of the NRF2 target genes catalase and SOD1 (Figure 9B). In addition, there was a very significant reduction in RSV-induced lung oxidative stress in BHA-treated mice, as indicated by a significant reduction of 8-isoprostane levels in BALs, compared to untreated, infected mice (Figure 9C), supporting our previous findings that BHA treatment has a positive impact on RSVinduced lung disease.

\section{DISCUSSION}

Since its isolation, RSV has been identified as a leading cause of epidemic respiratory infections in infants and children worldwide [2]. No efficacious treatment or vaccine yet exists for RSV and immunity is incomplete, resulting in repeated attacks of acute respiratory tract illness through adulthood [2]. Several recent studies have directly or indirectly indicated an important role of ROS produced by epithelial and inflammatory cells, and subsequent oxidative stress, in the pathogenesis of acute and chronic lung inflammatory diseases such as acute respiratory distress, cystic fibrosis, asthma and COPD [29-32]. We and others have shown that infection with RSV, the recently identified human metapneumovirus (hMPV), and influenza can all induce ROS formation [7, 33-36] and that inhibiting ROS production by administering antioxidants or recombinant SODs significantly decreases lung injury and improves clinical disease in RSV- and influenza-infected animals, suggesting that ROS play a significant role in the pathogenesis of virus-induced pneumonia [37, 38]. Although increased antioxidant defenses have been reported in certain pulmonary diseases resulting from exposure to hyperoxia [39], ozone [40] and cigarette smoke [41], our recent studies and data presented here show that RSV infection induces 
a significant decrease in the expression of most AOEs involved in maintaining the cellular oxidant-antioxidant balance, leading to cellular oxidative stress, both in vitro and in vivo . AOE gene transcription is regulated through binding of NRF2 to the ARE site located in the gene promoters [10]. Several viruses have been shown to induce ARE-dependent responses by activating NRF2. Among them, hepatitis B and C viruses, human cytomegalovirus and the Kaposi's sarcoma-associated herpes virus, which can all induce ROS formation (Reviewed in [34]), have been shown to activate NRF2 in infected cells, leading to the induction of cytoprotective genes, as a mechanism to protect infected cells from oxidative damage [42-46]. Similarly, Marburg virus, an important cause of human hemorrhagic fever, blocks KEAP1 activation, leading to the expression of AOE genes, to ensure survival of infected cells [47]. In our study, we found that RSV infection of AECs induces a transient NRF2 activation, demonstrated by increased NRF2 binding to the ARE of the AOE gene promoter and activation of ARE-dependent gene transcription at $6 \mathrm{~h}$ p.i., followed however by a progressive decrease in NRF2 activation, starting at 15h p.i., to levels below the ones found in uninfected cells (Figure 1), with a kinetics that mirror the progressive decrease in AOE expression observed in RSVinfected cells [4].

Reduced nuclear levels of NRF2 can occur as a result of various mechanisms, including decreased expression, increased degradation or through increased nuclear export [9]. Our results show that RSV infection is associated with increased NRF2 ubiquitination and degradation through a proteasomal pathway, based on our observations that the proteasomal inhibitors MG132 and Lactacystin rescue NRF2 expression and binding to the ARE site of the AOE gene promoters, restoring ARE-dependent gene transcription and AOE gene expression to that of uninfected cells (Figure 2 and 3). NRF2 degradation through the proteasome pathway occurred 
also in vivo, as MG132 treatment in mice was able to restore NRF2 nuclear levels in lungs of mice infected with RSV, however it had a modest impact on rescuing AOE gene expression, in particular on catalase (Figure 7). A possible explanation of these findings is that MG132 affects activation of other signaling molecules which in turn could be important in regulating AOE gene

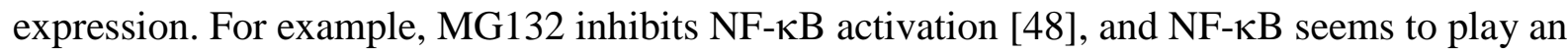
important role in transcriptional response to oxidative stress, including the expression of catalase and glutathione peroxidase [49]. Although stabilization of NRF2 by proteasome inhibition and subsequent transcriptional activation of its downstream genes, by preventing NRF2 degradation, have been shown in different cell types and disease conditions (Reviewed in [50]), suggesting that proteasome inhibition could be a promising therapeutic strategy for oxidative stress damageassociated diseases, it does not seem to have a beneficial effect in the context of RSV, at least in a mouse model of infection [51]. Treatment of RSV infected mice with bortezomib, an FDA approved proteasome inhibitor, resulted in increased pulmonary inflammation and disease, compared to untreated, infected animals. Whether NRF2 ubiquitination in response to RSV infection occurs through KEAP1, it remains to be established, as we observed it both in SAE and A549 cells, which carries a KEAP1 mutation that greatly reduces its repressor activity [52].

RSV-induced decrease in NRF2 activation could be rescued by treatment of AECs with the NRF2 inducer tBHQ, shown by restoration of NRF2 nuclear levels, ARE-dependent gene transcription and AOE expression, which resulted in a significant decrease of cellular oxidative stress (Figure 8). Administration of tBHQ was also able to rescue NRF2 activation in vivo, as indicated by a significant increase of NRF2 nuclear levels in lung extracts of RSV-infected mice, which were dramatically decreased by the infection (Figure 9), as we have previously described [5]. The NRF2-ARE pathway has been shown to play a protective role in the murine airways 
against RSV-induced injury and oxidative stress. More severe RSV disease, including higher viral titers, augmented inflammation, and enhanced mucus production and epithelial injury were found in $N R F 2-/-$ mice compared to $N R F 2+/+$ mice [53]. Similarly, lack of NRF2 expression resulted in increased influenza virus replication [54], while treatment of AECs with the NRF2 inducer sulforaphane or NRF2 overexpression led to significant inhibition of viral replication and oxidative stress $[54,55]$.

Post-translational modifications, such as phosphorylation and acetylation, are important regulators of transcription factor activation, regulating multiple steps of activation, from nuclear translocation, to DNA-binding to transcriptional activity. NRF2 has been shown to be acetylated by p300/CBP [56]. Acetylation promotes DNA binding of NRF2 and enhances gene transcription, although not of all NRF2 target genes [56]. It also regulates NRF2 cellular distribution, as deacetylating conditions results in relocalization of NRF2 to the cytoplasmic compartment [12]. As a dynamic and reversible process, acetylation of NRF2 is determined by the relative activities of HATs and histone deacetylases. Our results show that RSV infection was associated with increased deacetylase activity and reduced recruitment of CBP to the ARE site of AOE gene promoters, resulting in NRF2 deacetylation both in vitro and in vivo (Figure 4 and 7). CBP overexpression could rescue ARE-dependent gene transcription and treatment of infected cells with the HDAC inhibitor TSA led to restoration of NRF2 acetylation, suggesting that RSV infection is associated with an unbalance acetylation/deacetylation environment at the ARE transcription sites. HDAC inhibition was able to restore NRF2 nuclear levels and NRF2 binding to the ARE site of AOE gene promoters, therefore restoring ARE-dependent gene transcription and gene expression in RSV-infected cells (Figure 5). Importantly, HDAC inhibition was also associated with increased NRF2 cellular levels (Figure 5), suggesting that 
blocking RSV-induced NRF2 deacetylation might indeed protect NRF2 against degradation by retaining it in the nucleus, bound to its cognate promoter site. Although HDAC class III (sirtuins) have been shown to play a role in NRF2 deacetylation [12], we could not demonstrate a significant role of this class of HDAC in RSV-induced inhibition of NRF2 activation. On the other hand, class I HDAC1 and 2 seem to be important in regulating NRF2 function in infected cells, as inhibition of their expression was associated with restoration of NRF2 nuclear levels and NRF2-dependent gene expression both in A549 and SAECs (Figure 6). Although we did observe an increase in HDAC activity, there was no induction of HDAC 1 or 2 expression in RSVinfected cells (Supplementary Figure 4). Induction of global HDAC activity has been reported in other disease models, such as cardiac hypertrophy and ischemia-reperfusion injury, as well as rheumathoid arthritis $[57,58]$. HDAC1 and 2 activity is regulated at multiple levels (Reviewed in [59]). Both proteins are active within a complex of proteins, the better characterized being Sin3, NuRD (nucleosome remodelling and deacetylating) and Co-REST, which are necessary for modulating HDAC deacetylase activity and DNA-binding, together with other proteins that mediate the recruitment of HDACs to gene promoters. A second way of regulating HDAC activity is via post-translational modifications. Both activity and complex-formation are regulated by phosphorylation. HDAC1 and HDAC2 are phosphorylated at a low level in resting cells and hyperphosphorylation leads to a significant increase in deacetylase activity. It is possible that RSV infection leads to modification of either or both of these important regulatory elements of HDAC activation. HDAC inhibitors are currently being developed as a new class of anti-cancer agents, many of which have already entered clinical trials, and our findings suggest that they could represent an attractive novel treatment for viral-induced lung inflammation. 
In conclusion, RSV-induced respiratory disease is associated with increased ROS generation and oxidative stress that are likely to play a key role in initiating and amplifying lung injury and inflammation. Compounds that stimulate ARE-driven transcription, as well as possibly HDAC inhibitors, could hold great potential for modulating RSV-induced oxidative stress and the associated lung damage.

\section{ACKNOWLEDGMENTS}

This project was supported by R01 AI062885, R21 AI111042, R21 AI103565, P01 AI07924602, P30 ES006676 and W81XWH1010146-DoD. We would like to acknowledge Tianshuang Liu and Yinghong Ma for their technical assistance and Cynthia Tribble for manuscript editing and submission. 


\section{REFERENCES}

[1] Hall C.B., Weinberg G.A., Iwane M.K., Blumkin A.K., Edwards K.M., Staat M.A. et al. The burden of respiratory syncytial virus infection in young children. $N$ Engl J Med ;360:588-98;2009

[2] Hall C.B. Respiratory syncytial virus and parainfluenza virus. N Engl J Med ;344:1917$28 ; 2001$

[3] Falsey A.R., Hennessey P.A., Formica M.A., Cox C., Walsh E.E. Respiratory syncytial virus infection in elderly and high-risk adults. N Engl J Med ;352:1749-59;2005

[4] Hosakote Y.M., Liu T., Castro S.M., Garofalo R.P., Casola A. Respiratory syncytial virus induces oxidative stress by modulating antioxidant enzymes. Am J Respir Cell Mol Biol ;41:348-57;2009

[5] Hosakote Y.M., Jantzi P.D., Esham D.L., Spratt H., Kurosky A., Casola A. et al. Viralmediated inhibition of antioxidant enzymes contributes to the pathogenesis of severe respiratory syncytial virus bronchiolitis. Am J Respir Crit Care Med ;183:1550-60;2011

[6] Castro S.M., Guerrero-Plata A., Suarez-Real G., Adegboyega P.A., Colasurdo G.N., Khan A.M. et al. Antioxidant Treatment Ameliorates Respiratory Syncytial Virusinduced Disease and Lung Inflammation. Am J Respir Crit Care Med;174:1361-9;2006

[7] Casola A., Burger N., Liu T., Jamaluddin M., Brasier A.R., Garofalo R.P. Oxidant tone regulates RANTES gene transcription in airway epithelial cells infected with Respiratory Syncytial Virus: role in viral-induced Interferon Regulatory Factor activation. J Biol Chem ;276:19715-22;2001

[8] Liu T., Castro S., Brasier A.R., Jamaluddin M., Garofalo R.P., Casola A. Reactive oxygen species mediate virus-induced STAT activation: role of tyrosine phosphatases. $J$ Biol Chem ;279:2461-9;2004

[9] Kaspar J.W., Niture S.K., Jaiswal A.K. Nrf2:INrf2 (Keap1) signaling in oxidative stress. Free Radic Biol Med;47:1304-9;2009

[10] Jaiswal A.K. Nrf2 signaling in coordinated activation of antioxidant gene expression. Free Radic Biol Med ;36:1199-207;2004

[11] Bloom D.A., Jaiswal A.K. Phosphorylation of Nrf2 at Ser40 by protein kinase C in response to antioxidants leads to the release of $\mathrm{Nrf} 2$ from INrf2, but is not required for Nrf2 stabilization/accumulation in the nucleus and transcriptional activation of antioxidant response element-mediated $\mathrm{NAD}(\mathrm{P}) \mathrm{H}$ :quinone oxidoreductase-1 gene expression. J Biol Chem ;278:44675-82;2003 
[12] Kawai Y., Garduno L., Theodore M., Yang J., Arinze I.J. Acetylation-deacetylation of the transcription factor Nrf2 (nuclear factor erythroid 2-related factor 2) regulates its transcriptional activity and nucleocytoplasmic localization. J Biol Chem ;286:7629$40 ; 2011$

[13] Ueba O. Respiratory syncytial virus: I. concentration and purification of the infectious virus. Acta Med Okayama;32:265-72;1978

[14] Patel J.A., Kunimoto M., Sim T.C., Garofalo R., Eliott T., Baron S. et al. Interleukin-1 alpha mediates the enhanced expression of intercellular adhesion molecule- 1 in pulmonary epithelial cells infected with respiratory syncytial virus. Am J Resp Cell Mol ;13:602-9;1995

[15] Casola A., Garofalo R.P., Jamaluddin M., Vlahopoulos S., Brasier A.R. Requirement of a novel upstream response element in RSV induction of interleukin-8 gene expression: stimulus-specific differences with cytokine activation. J Immunol ;164:5944-51;2000

[16] Schreiber E., Matthias P., Muller M.M., Schaffner W. Rapid detection of octamer binding proteins with 'mini-extracts', prepared from a small number of cells. Nucleic Acids Res ; 17:6419;1989

[17] Bao X., Liu T., Shan Y., Li K., Garofalo R.P., Casola A. Human metapneumovirus glycoprotein G inhibits innate immune responses. PLoS Pathog;4:e1000077;2008

[18] Welliver T.P., Garofalo R.P., Hosakote Y., Hintz K.H., Avendano L., Sanchez K. et al. Severe human lower respiratory tract illness caused by respiratory syncytial virus and influenza virus is characterized by the absence of pulmonary cytotoxic lymphocyte responses. J Infect Dis ;195:1126-36;2007

[19] Johnson J.E., Gonzales R.A., Olson S.J., Wright P.F., Graham B.S. The histopathology of fatal untreated human respiratory syncytial virus infection. Mod Pathol;20:108-19;2007

[20] Gregoretti I.V., Lee Y.M., Goodson H.V. Molecular evolution of the histone deacetylase family: functional implications of phylogenetic analysis. J Mol Biol ;338:17-31;2004

[21] Dokmanovic M., Clarke C., Marks P.A. Histone deacetylase inhibitors: overview and perspectives. Mol Cancer Res ;5:981-9;2007

[22] Gertz M., Fischer F., Nguyen G.T., Lakshminarasimhan M., Schutkowski M., Weyand M. et al. Ex-527 inhibits Sirtuins by exploiting their unique NAD+-dependent deacetylation mechanism. Proc Natl Acad Sci U S A ;110:E2772-E2781;2013

[23] Verdin E., Dequiedt F., Kasler H.G. Class II histone deacetylases: versatile regulators. Trends Genet ;19:286-93;2003

[24] Yang X.J., Gregoire S. Class II histone deacetylases: from sequence to function, regulation, and clinical implication. Mol Cell Biol ;25:2873-84;2005 
[25] Spange S., Wagner T., Heinzel T., Kramer O.H. Acetylation of non-histone proteins modulates cellular signalling at multiple levels. Int J Biochem Cell Biol ;41:185-98;2009

[26] Khochbin S., Verdel A., Lemercier C., Seigneurin-Berny D. Functional significance of histone deacetylase diversity. Curr Opin Genet Dev ;11:162-6;2001

[27] Hur W., Gray N.S. Small molecule modulators of antioxidant response pathway. Curr Opin Chem Biol ;15:162-73;2011

[28] Keum Y.S., Han Y.H., Liew C., Kim J.H., Xu C., Yuan X. et al. Induction of heme oxygenase-1 (HO-1) and NAD[P]H: quinone oxidoreductase 1 (NQO1) by a phenolic antioxidant, butylated hydroxyanisole (BHA) and its metabolite, tert-butylhydroquinone (tBHQ) in primary-cultured human and rat hepatocytes. Pharm Res ;23:2586-94;2006

[29] Lucidi V., Ciabattoni G., Bella S., Barnes P.J., Montuschi P. Exhaled 8-isoprostane and prostaglandin $\mathrm{E}(2)$ in patients with stable and unstable cystic fibrosis. Free Radic Biol Med ;45:913-9;2008

[30] MacNee W. Oxidative stress and lung inflammation in airways disease. Eur J Pharmacol ;429:195-207;2001

[31] Rahman I., Morrison D., Donaldson K., MacNee W. Systemic oxidative stress in asthma, COPD, and smokers. Am J Respir Crit Care Med ;154:1055-60;1996

[32] Morcillo E.J., Estrela J., Cortijo J. Oxidative stress and pulmonary inflammation: pharmacological intervention with antioxidants. Pharmacol Res;40:393-404;1999

[33] Bao X., Sinha M., Liu T., Hong C., Luxon B.A., Garofalo R.P. et al. Identification of human metapneumovirus-induced gene networks in airway epithelial cells by microarray analysis. Virology ;374:114-27;2008

[34] Choi A.M., Knobil K., Otterbein S.L., Eastman D.A., Jacoby D.B. Oxidant stress responses in influenza virus pneumonia: gene expression and transcription factor activation. Am J Physiol ;271:L383-L391;1996

[35] Jacoby D.B., Choi A.M. Influenza virus induces expression of antioxidant genes in human epithelial cells. Free Radic Biol Med ;16:821-4;1994

[36] Jamaluddin M., Tian B., Boldogh I., Garofalo R.P., Brasier A.R. Respiratory syncytial virus infection induces a reactive oxygen species-MSK1-phospho-Ser-276 RelA pathway required for cytokine expression. J Virol ;83:10605-15;2009

[37] Akaike T., Ando M., Oda T., Doi T., Ljiri S., Araki S. et al. Dependence on O2 generation by xanthine oxidase of pathogenesis of influenza virus infection in mice. $J$ Clin Invest $; \mathbf{8 5} ; 1990$ 
[38] Akaike T., Noguchi Y., Ijiri S., Setoguchi K., Suga M., Zheng Y.M. et al. Pathogenesis of influenza virus-induced pneumonia: involvement of both nitric oxide and oxygen radicals. Proc Natl Acad Sci U S A ;93:2448-53;1996

[39] Erzurum S.C., Danel C., Gillissen A., Chu C.S., Trapnell B.C., Crystal R.G. In vivo antioxidant gene expression in human airway epithelium of normal individuals exposed to $100 \%$ O2. J Appl Physiol ;75:1256-62;1993

[40] Boehme D.S., Hotchkiss J.A., Henderson R.F. Glutathione and GSH-dependent enzymes in bronchoalveolar lavage fluid cells in response to ozone. Exp Mol Pathol ;56:37$48 ; 1992$

[41] Gilks C.B., Price K., Wright J.L., Churg A. Antioxidant gene expression in rat lung after exposure to cigarette smoke. Am J Pathol ;152:269-78;1998

[42] Schaedler S., Krause J., Himmelsbach K., Carvajal-Yepes M., Lieder F., Klingel K. et al. Hepatitis B virus induces expression of antioxidant response element-regulated genes by activation of Nrf2. J Biol Chem ;285:41074-86;2010

[43] Burdette D., Olivarez M., Waris G. Activation of transcription factor Nrf2 by hepatitis C virus induces the cell-survival pathway. J Gen Virol ;91:681-90;2010

[44] Ivanov A.V., Smirnova O.A., Ivanova O.N., Masalova O.V., Kochetkov S.N., Isaguliants M.G. Hepatitis $\mathrm{C}$ virus proteins activate NRF2/ARE pathway by distinct ROS-dependent and independent mechanisms in HUH7 cells. PLoS ONE ;6:e24957;2011

[45] Lee J., Koh K., Kim Y.E., Ahn J.H., Kim S. Up-regulation of Nrf2 Expression by Human Cytomegalovirus Infection Protects Host Cells from Oxidative Stress. J Gen Virol ;94:1658-68;2013

[46] Gjyshi O., Bottero V., Veettil M.V., Dutta S., Singh V.V., Chikoti L. et al. Kaposi's sarcoma-associated herpesvirus induces Nrf2 during de novo infection of endothelial cells to create a microenvironment conducive to infection. PLoS Pathog ;10:e1004460;2014

[47] Page A., Volchkova V.A., Reid S.P., Mateo M., Bagnaud-Baule A., Nemirov K. et al. Marburgvirus Hijacks Nrf2-Dependent Pathway by Targeting Nrf2-Negative Regulator Keap1. Cell Rep ;2014

[48] Karin M., Delhase M. The I kappa B kinase (IKK) and NF-kappa B: key elements of proinflammatory signalling. Semin Immunol ;12:85-98;2000

[49] Zhou L.Z., Johnson A.P., Rando T.A. NF kappa B and AP-1 mediate transcriptional responses to oxidative stress in skeletal muscle cells. Free Radic Biol Med ;31:1405$16 ; 2001$ 
[50] Cui W., Bai Y., Luo P., Miao L., Cai L. Preventive and therapeutic effects of MG132 by activating Nrf2-ARE signaling pathway on oxidative stress-induced cardiovascular and renal injury. Oxid Med Cell Longev ;2013:306073;2013

[51] Lupfer C., Patton K.M., Pastey M.K. Treatment of human respiratory syncytial virus infected Balb/C mice with the proteasome inhibitor bortezomib (Velcade, PS-341) results in increased inflammation and mortality. Toxicology ;268:25-30;2010

[52] Singh A., Misra V., Thimmulappa R.K., Lee H., Ames S., Hoque M.O. et al. Dysfunctional KEAP1-NRF2 interaction in non-small-cell lung cancer. PLoS Med ;3:e420;2006

[53] Cho H.Y., Imani F., Miller-Degraff L., Walters D., Melendi G.A., Yamamoto M. et al. Antiviral Activity of Nrf2 in a Murine Model of Respiratory Syncytial Virus (RSV) Disease. Am J Respir Crit Care Med ;2008

[54] Kesic M.J., Simmons S.O., Bauer R., Jaspers I. Nrf2 expression modifies influenza A entry and replication in nasal epithelial cells. Free Radic Biol Med ;51:444-53;2011

[55] Kosmider B., Messier E.M., Janssen W.J., Nahreini P., Wang J., Hartshorn K.L. et al. Nrf2 protects human alveolar epithelial cells against injury induced by influenza A virus. Respir Res ;13:43;2012

[56] Sun Z., Chin Y.E., Zhang D.D. Acetylation of Nrf2 by p300/CBP augments promoterspecific DNA binding of Nrf2 during the antioxidant response. Mol Cell Biol;29:2658$72 ; 2009$

[57] McKinsey T.A. Therapeutic potential for HDAC inhibitors in the heart. Annu Rev Pharmacol Toxicol ;52:303-19;2012

[58] Gillespie J., Savic S., Wong C., Hempshall A., Inman M., Emery P. et al. Histone deacetylases are dysregulated in rheumatoid arthritis and a novel histone deacetylase 3selective inhibitor reduces interleukin- 6 production by peripheral blood mononuclear cells from rheumatoid arthritis patients. Arthritis Rheum ;64:418-22;2012

[59] de Ruijter A.J., van Gennip A.H., Caron H.N., Kemp S., van Kuilenburg A.B. Histone deacetylases (HDACs): characterization of the classical HDAC family. Biochem J ;370:737-49;2003 


\section{FIGURE LEGENDS}

\section{Figure 1. RSV infection down-regulates NRF2 dependent gene transcription}

(A) Nuclear proteins isolated from A549 cells (left panel) and SAECs (right panel) uninfected or infected with RSV for 6, 15 and 24h were subjected to Western blot analysis using anti-NRF2 antibody. For loading controls, membranes were stripped and re-probed with anti-Lamin B antibody. The blots are representative of three independent experiments. Densitometric analysis of NRF2 band intensity is shown after normalization to Lamin B. The groups were analyzed by one-way ANOVA followed by Tukey's post-hoc test. Data are shown as mean \pm SEM. $* P<0.05$ relative to uninfected cells, $* * P<0.05$ relative to uninfected and 6 h infected cells. Open bars represent uninfected (control, Ctrl) and solid bars represent RSV infected cells. (B) A549 cells were transiently transfected with an ARE driven luciferase reporter plasmid, infected with RSV for various lengths of time, and harvested to measure luciferase activity. Data are representative of three independent experiments. The groups were analyzed by one-way ANOVA followed by Tukey's post-hoc test. Data are shown as mean \pm SEM. ${ }^{*} P<0.05$ relative to uninfected cells, $* * P<0.05$ relative to uninfected and $6 \mathrm{~h}$ infected cells. (C) ChIP-QgPCR analysis of NRF2 occupancy of endogenous ARE promoter sites. Chromatin DNA from A549 cells uninfected or infected with RSV for 6, 15 and 24h was immunoprecipitated using anti-NRF2 antibody or IgG as negative control. QgPCR was performed using primers spanning the ARE binding site of the catalase (left) and SOD1 (right) gene promoter. Total input chromatin DNA for immunoprecipitation was included as positive control for QgPCR amplification. Fold change was calculated compared to $\operatorname{IgG}$ control. Data are representative of three independent experiments. The groups were analyzed by one-way ANOVA followed by Tukey's post-hoc test. 
Data are shown as mean \pm SEM $* P<0.05$ relative to uninfected cells, $* * P<0.05$ relative to uninfected and $6 \mathrm{~h}$ infected cells.

\section{Figure 2. RSV infection is associated with proteasome-dependent NRF2 degradation}

(A) Total cell lysates prepared from A549 cells (left panel) and SAECs (right panel) uninfected or infected with RSV for 6, 15 and 24h were subjected to Western blot analysis using anti-NRF2 antibody. For loading controls, membranes were stripped and reprobed using anti- $\beta$-Actin antibody. The blots are representative of three independent experiments. Densitometric analysis of NRF2 band intensity is shown after normalization to $\beta$-Actin. Open bars represent uninfected (Ctrl) and solid bars represent RSV infected cells. The groups were analyzed by one-way ANOVA followed by Tukey's post-hoc test. Data are shown as mean \pm SEM. $* P<0.05$ relative to uninfected cells, $* * P<0.05$ relative to uninfected and $6 \mathrm{~h}$ infected cells. (B) Total cell lysates prepared from A549 cells uninfected or infected with RSV for 18h in the presence or absence of $10 \mu \mathrm{M}$ Lactacystin (Lact) were subjected to Western blot analysis using anti-NRF2 antibody. For loading controls, membranes were stripped and reprobed with anti- $\beta$-Actin antibody. The blots are representative of three independent experiments. Densitometric analysis of NRF2 band intensity is shown after normalization to $\beta$-Actin. The groups were analyzed by two-way ANOVA followed by Bonferroni post-test. Data are shown as mean \pm SEM. ${ }^{*} P<0.05$ relative to untreated, RSV infected cells. (C) Total cell lysates prepared from A549 cells (left panel) and SAECs (right panel), uninfected or infected with RSV for 6 and 15h, were immunoprecipitated using anti-NRF2 antibody and immune complexes analyzed by Western blots using antiubiquitin antibody. Membranes were stripped and reprobed with anti-NRF2 antibody to determine the level of immunoprecipitated NRF2. Lower panel shows NRF2 Western blot of 
input proteins and $\beta$-Actin as internal control. Blots are representative of two independent experiments.

\section{Figure 3. Blocking NRF2 degradation rescues ARE-dependent gene expression}

(A) A549 cells were transiently transfected with the ARE-luciferase reporter plasmid. Cells uninfected and infected with RSV for $18 \mathrm{~h}$ in the presence or absence of either $10 \mu \mathrm{M}$ Lactacystin or MG132 were harvested to measure luciferase activity. Data are representative of three independent experiments. The groups were analyzed by two-way ANOVA followed by Bonferroni post-test. Data are shown as mean \pm SEM. $* P<0.05$ relative to RSV infected, untreated cells. (B) A549 cells, uninfected or infected with RSV for $18 \mathrm{~h}$ in the presence or absence of either $10 \mu \mathrm{M}$ Lactacystin or MG132, were harvested to prepare total RNA. NQO1, Catalase, SOD1, GPX1 and GCLC gene expression were quantified by real-time PCR. Data are representative of three independent experiments. The groups were analyzed by two-way ANOVA followed by Bonferroni post-test. Data are shown as mean \pm SEM. $* P<0.05$ relative to RSV infected, untreated cells. (C) Chromatin DNA from A549 cells uninfected or infected with RSV for $18 \mathrm{~h}$ in the presence or absence of either $10 \mu \mathrm{M}$ Lactacystin or MG132 was immunoprecipitated using anti-NRF2 antibody or IgG as negative control. QgPCR was performed using primers spanning the ARE binding site of the catalase (left panel) or SOD1 (right panel) gene promoter. Total input chromatin DNA for immunoprecipitation was included as positive control for QgPCR amplification. Fold change was calculated compared to IgG control. Data are representative of three independent experiments. The groups were analyzed by one-way ANOVA followed by Tukey's post-hoc test. Data are shown as mean \pm SEM. $* P<0.05$ relative to untreated, RSV infected cells. 


\section{Figure 4. RSV infection induces NRF2 deacetylation}

(A) Total cell lysates from A549 (left panel) and SAECs (right panel) uninfected or infected with RSV for $15 \mathrm{~h}$ in the presence or absence of $250 \mathrm{nM}$ TSA were immunoprecipitated using antiNRF2 antibody and subjected to Western blot using anti-acetyl lysine antibody. Lower panel shows NRF2 Western blot for input of the IP. $\beta$-Actin was used as loading control. (B) HDAC activity in nuclear extracts prepared from A549 (left panel) and SAECs (right panel) uninfected and infected with RSV for 6, 15 and 24h was analyzed by using HDAC activity assay kit (Cayman). Data are representative of three independent experiments. The groups were analyzed by one-way ANOVA followed by Tukey's post-hoc test. Data are shown as mean $\pm \mathrm{SEM} .{ }^{*} P<$ 0.05 relative to uninfected cells. (C) Chromatin DNA from A549 cells uninfected or infected with RSV for 6,15 , and $24 \mathrm{~h}$ was immunoprecipitated using anti-CBP antibody or IgG as negative control. QgPCR was performed using primers spanning the ARE binding site of the SOD1 promoter. Total input chromatin DNA for immunoprecipitation was included as positive control for QgPCR amplification. Fold change was calculated compared to IgG control. Data are representative of three independent experiments. The groups were analyzed by one-way ANOVA followed by Tukey's post-hoc test. Data are shown as mean \pm SEM. ${ }^{*} P<0.05$ relative to uninfected cells, ${ }^{* *} P<0.05$ relative to uninfected and $6 \mathrm{~h}$ infected cells. (D) A549 cells, transiently co-transfected with the ARE-luciferase reporter plasmid and CBP expression plasmid or empty vector (EV), were infected with RSV for $18 \mathrm{~h}$ and harvested to measure luciferase activity. Data are representative of three independent experiments. The groups were analyzed by one-way ANOVA followed by Tukey's post-hoc test. Data are shown as mean \pm SEM. $* P<0.05$ relative to EV transfected, RSV infected cells. 


\section{Figure 5. Blocking HDAC activity rescues NRF2-dependent gene transcription}

Nuclear protein $(\mathbf{A})$ or total cell lysates $(\mathbf{B})$ were prepared from A549 cells (left panel) and SAECs (right panel) uninfected or infected with RSV for $18 \mathrm{~h}$ in the presence or absence of 250 nM TSA were subjected to Western blot analysis using anti-NRF2 antibody. For loading controls, membranes were stripped and reprobed using either anti-Lamin B or $\beta$-Actin antibody. The blots are representative of three independent experiments. Densitometric analysis of NRF2 band intensity is shown after normalization to the appropriate internal control. The groups were analyzed by two-way ANOVA followed by Bonferroni post-test. Data are shown as mean \pm SEM. ${ }^{*} P<0.05$ relative to untreated, RSV infected cells. (C) A549 cells were transiently transfected with the ARE-luciferase reporter plasmid. Cells uninfected or infected with RSV for $18 \mathrm{~h}$ in the presence or absence of $250 \mathrm{nM}$ TSA were harvested to measure luciferase activity. The groups were analyzed by two-way ANOVA followed by Bonferroni post-test. Data are shown as mean \pm SEM. ${ }^{*} P<0.05$ relative to untreated, RSV infected cells. (D) A549 cells (upper panel) and SAECs (lower panel) uninfected or infected with RSV for $18 \mathrm{~h}$ in the presence or absence of $250 \mathrm{nM}$ TSA were harvested to prepare total RNA. Catalase and SOD1 gene expression was quantified by real-time PCR. Data are representative of three independent experiments. The groups were analyzed by two-way ANOVA followed by Bonferroni post-test. Data are shown as mean \pm SEM. ${ }^{*} P<0.05$ relative to untreated, RSV infected cells. $(\mathbf{E})$ Chromatin DNA from A549 cells uninfected or infected with RSV for 6, 15, and 24h in the presence or absence of $250 \mathrm{nM}$ TSA was immunoprecipitated using an anti-NRF2 antibody (or IgG as negative control. QgPCR was performed using primers spanning the ARE binding site of the catalase (left) and SOD1 (right) gene promoters. Total input chromatin DNA for 
immunoprecipitation was included as positive control for QgPCR amplification. Fold change was calculated compared to IgG control. Data are representative of three independent experiments. The groups were analyzed by one-way ANOVA followed by Tukey's post-hoc test. Data are shown as mean \pm SEM. $* P<0.05$ relative to untreated, RSV infected cells.

\section{Figure 6. Blocking HDAC1 and 2 expression rescues NRF2 activation}

Nuclear protein prepared from A549 cells transfected with nontarget siRNA or (A) HDAC1 or (B) HDAC2 or (C) HDAC3 siRNA, uninfected or infected with RSV for 18h, were subjected to Western blot analysis with anti-NRF2 antibody. Membranes were stripped and reprobed with anti-HDAC1/2/3 and anti-Lamin B antibodies for loading control. The blots are representative of three independent experiments. Densitometric analysis of NRF2 band intensity is shown after normalization to Lamin B. The groups were analyzed by two-way ANOVA followed by Bonferroni post-test. Data are shown as mean \pm SEM. $* P<0.05$ relative to nontarget siRNA transfected, RSV infected cells. (D) A549 cells transfected with nontarget siRNA or siRNAs for HDAC1, 2 or 3, uninfected or infected with RSV for 18h, were harvested to prepare total RNA. Catalase (left panel) and SOD1 (right panel) gene expression was quantified by real-time PCR. Data are representative of three independent experiments. The groups were analyzed by two-way ANOVA followed by Bonferroni post-test. Data are shown as mean \pm SEM. $* P<0.05$ relative to nontarget siRNA transfected, RSV infected cells. (E) Chromatin DNA from A549 cells uninfected or infected with RSV for 6 and $15 \mathrm{~h}$ in the presence or absence of $250 \mathrm{nM}$ TSA was immunoprecipitated using anti-HDAC1 antibody or IgG as negative control. QgPCR was performed using primers spanning the ARE binding site of the SOD1 promoter. Total input chromatin DNA for immunoprecipitation was included as positive control for QgPCR 
amplification. Fold change was calculated compared to IgG control. Data are representative of three independent experiments. The groups were analyzed by one-way ANOVA followed by Tukey's post-hoc test. Data are shown as mean \pm SEM. ${ }^{*} P<0.05$ relative to uninfected cells, ** $P<0.05$ relative to untreated, RSV infected cells.

\section{Figure 7. NRF2 is deacetylated and degraded through the proteasome pathway in vivo}

Nuclear protein isolated from lungs of mice that were either sham inoculated (S1-S3) or infected with RSV (R1-R3) for 48h were (A) analyzed for HDAC activity by using HDAC activity assay kit. Data are representative of three independent experiments. The groups were analyzed by oneway ANOVA followed by Tukey's post-hoc test. Data are shown as mean \pm SEM. ${ }^{*} P<0.05$ relative to sham inoculated mice. (B) immunoprecipitated using anti-NRF2 antibody and subjected to Western blot using anti-acetyl lysine antibody. Lower panel shows NRF2 Western blot for input of the IP. Lamin B was used as loading control. (C) Nuclear protein prepared from lungs of mice that were either sham inoculated or infected with RSV for $48 \mathrm{~h}$ in the presence or absence of MG132 were subjected to Western blot analysis using anti-NRF2 antibody. S1-S3: sham inoculated mice, M1- M3: Sham inoculated, MG132 treated mice, R1-R3: RSV infected mice, and MR1-MR3: MG132 treated, RSV infected mice. For loading controls, membranes were stripped and re-probed with anti-Lamin B antibody. The blots are representative of three independent experiments. Densitometric analysis of NRF2 band intensity is shown after

normalization to Lamin B. The groups were analyzed by two-way ANOVA followed by Bonferroni post-test. Data are shown as mean \pm SEM. $* P<0.05$ relative to untreated, RSV infected mice. (D) Catalase (left panel) and SOD1 (right panel) gene expression was quantified by q-RT-PCR. Data are representative of three independent experiments. The groups were 
analyzed by two-way ANOVA followed by Bonferroni post-test. Data are shown as mean \pm SEM. ${ }^{*} P<0.05$ relative to untreated, RSV infected mice.

\section{Figure 8. NRF2 modulation rescues ARE-dependent gene transcription and ameliorates oxidative stress during RSV infection}

(A) A549 cells were transiently transfected with the ARE-luciferase reporter plasmid, uninfected or infected with RSV for $18 \mathrm{~h}$ in the presence or absence of $25 \mu \mathrm{M} \mathrm{tBHQ}$, and harvested to measure luciferase activity. Data are representative of three independent experiments. The groups were analyzed by two-way ANOVA followed by Bonferroni post-test. Data are shown as mean \pm SEM. ${ }^{*} P<0.05$ relative to untreated, RSV infected cells. (B) SAECs, uninfected or infected with RSV for $18 \mathrm{~h}$ in the presence or absence of $25 \mu \mathrm{M}$ tBHQ, were harvested to prepare total RNA. Catalase (left panel) and SOD1 (right panel) gene expression was quantified by realtime PCR. Data are presented as fold changes and are representative of three independent experiments. The groups were analyzed by two-way ANOVA followed by Bonferroni post-test. Data are shown as mean \pm SEM. ${ }^{*} P<0.05$ relative to untreated, RSV infected cells. (C) SAECs were infected with RSV for $18 \mathrm{~h}$ in the presence or absence of $25 \mu \mathrm{M}$ tBHQ. Nuclear protein and total cell lysates were subjected to Western blot analysis using anti NRF2 or SOD1 antibodies. For loading controls, membranes were stripped and reprobed using anti Lamin B antibody for nuclear fractions or anti $\beta$-Actin antibody for total cell lysates. The blots are representative of three independent experiments. Densitometric analysis of NRF2 and SOD1 band intensity is shown after normalization to the appropriate internal control. The groups were analyzed by twoway ANOVA followed by Bonferroni post-test. Data are shown as mean \pm SEM. ${ }^{*} P<0.05$ relative to untreated, RSV infected cells. (D) Oxidative stress marker 8-isoprostane was 
measured by competitive enzyme immunoassay from the supernatant of SAECs uninfected or infected with RSV for $18 \mathrm{~h}$ in the presence or absence of $25 \mu \mathrm{M}$ tBHQ. Data are representative of three independent experiments. The groups were analyzed by two-way ANOVA followed by Bonferroni post-test. Data are shown as mean \pm SEM. $* P<0.05$ relative to untreated, RSV infected cells. (E) HDAC activity in nuclear extracts prepared from SAECs uninfected and infected with RSV for $18 \mathrm{~h}$ in the presence or absence of $25 \mu \mathrm{M}$ tBHQ were analyzed by using HDAC activity assay kit. Data are representative of three independent experiments. The groups were analyzed by two-way ANOVA followed by Bonferroni post-test. Data are shown as mean \pm SEM.

Figure 9. BHA treatment rescues NRF2 expression and ameliorates oxidative stress in vivo during RSV infection

(A) Nuclear protein isolated from lungs of mice that were either sham inoculated or infected with RSV for $48 \mathrm{~h}$ in presence or absence of BHA were subjected to Western blot analysis with antiNRF2 antibody. S1-S4: sham inoculated mice, R1-R4: RSV infected mice and B1-B4: BHA treated and RSV infected mice. For loading controls, membranes were stripped and reprobed with anti-Lamin B antibody. The blots are representative of three independent experiments. Densitometric analysis of NRF2 band intensity is shown after normalization to Lamin B. The groups were analyzed by one-way ANOVA followed by Tukey's post-hoc test. Data are shown as mean \pm SEM. ${ }^{*} P<0.05$ relative to untreated, RSV infected mice. (B) Catalase (left panel) and SOD1 (right panel) gene expression was quantified by q-RT-PCR. Data are representative of three independent experiments. The groups were analyzed by one-way ANOVA followed by Tukey's post-hoc test. Data are shown as mean \pm SEM. ${ }^{*} P<0.05$ relative to untreated, RSV 
infected mice. (C) Oxidative stress marker 8-isoprostane was measured by competitive enzyme immunoassay in BAL of mice sham inoculated or infected with RSV for 48h in the presence or absence of BHA. Data are representative of three independent experiments. The groups were analyzed by one-way ANOVA followed by Tukey's post-hoc test. Data are shown as mean \pm SEM. $* P<0.05$ relative to untreated, RSV infected mice. 


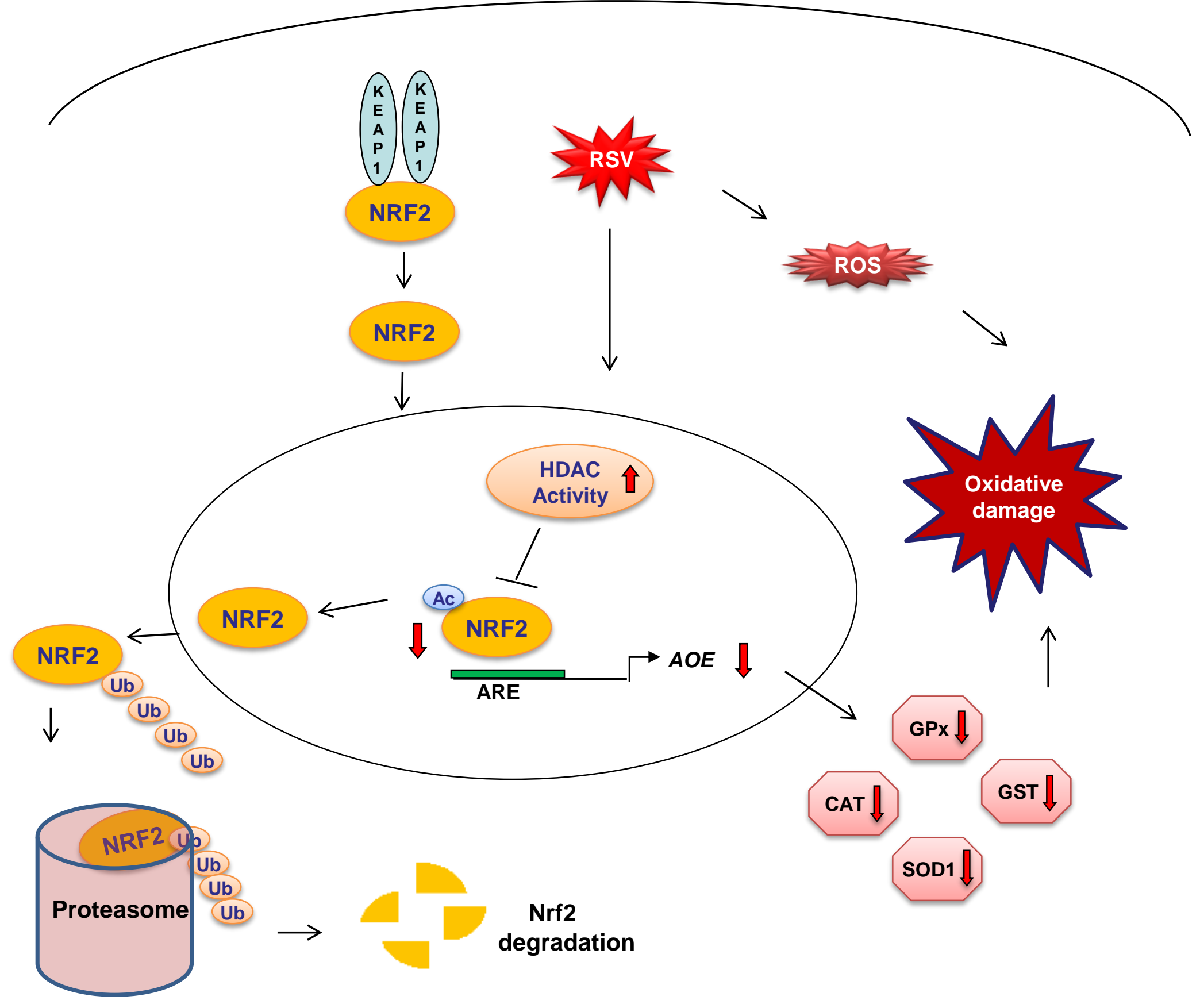

